\title{
On the interaction of a planar shock with a light polygonal interface
}

\author{
Zhigang Zhai ${ }^{1}$, Minghu Wang ${ }^{1}$, Ting $\mathrm{Si}^{1}$ and Xisheng Luo ${ }^{1,2, \dagger}$ \\ ${ }^{1}$ Advanced Propulsion Laboratory, Department of Modern Mechanics, \\ University of Science and Technology of China, Hefei 230026, China \\ ${ }^{2}$ State Key Laboratory of Fire Science, University of Science and Technology of China, \\ Hefei 230026, China
}

(Received 9 May 2014; revised 30 August 2014; accepted 3 September 2014;

first published online 26 September 2014)

The interaction of a planar shock wave with a polygonal $\mathrm{N}_{2}$ volume surrounded by $\mathrm{SF}_{6}$ is investigated experimentally and numerically. Three polygonal interfaces (square, triangle and diamond) are formed by the soap film technique developed in our previous work, in which thin pins are introduced as angular vertexes to connect adjacent sides of polygonal soap films. The evolutions of the shock-accelerated polygonal interfaces are then visualized by a high-speed schlieren system. Wave systems and interface structures can be clearly identified in experimental schlieren images, and agree well with the numerical ones. Quantitatively, the movement of the distorted interface, and the length and height of the interface structures are further compared and good agreements are achieved between experimental and numerical results. It is found that the evolution of these polygonal interfaces is closely related to their initial shapes. In the square interface, two vortices are generated shortly after the shock impact around the left corner and dominate the flow field at late stages. In the triangular and diamond cases, the most remarkable feature is the small ' $\mathrm{SF}_{6}$ jet' which grows constantly with time and penetrates the downstream boundary of the interface, forming two independent vortices. These distinct morphologies of the three polygonal interfaces also lead to the different behaviours of the interface features including the length and height. It is also found that the velocities of the vortex pair predicted from the theory of Rudinger and Somers (J. Fluid Mech., vol. 7, 1960, pp. 161-176) agree with the experimental ones, especially for the square case. Typical free precursor irregular refraction phenomena and the transitions among them are observed and analysed, which gives direct experimental evidence for wave patterns and their transitions at a slow/fast interface. The velocities of triple points and shocks are experimentally measured. It is found that the transmitted shock near the interface boundary has weakened into an evanescent wave.

Key words: compressible flows, mixing, shock waves 


\section{Introduction}

When a shock wave passes through a density inhomogeneity, the strong coupling of several types of fluid dynamics phenomena, including shock-wave refraction and reflection, vorticity production and transport, and turbulence, is involved. A complex wave pattern results from the shock refraction at a gaseous interface, which could broadly be classified into regular and irregular systems. Jahn (1956) experimentally studied the refraction of planar shock waves at air/ $\mathrm{CH}_{4}$ and air/ $\mathrm{CO}_{2}$ interfaces, and multi-phenomena of shock refraction were observed. Subsequently, Henderson (1966) numerically dealt with the regular refraction. Later, extensive examinations of a planar shock with different strengths refracting at either a slow/fast or a fast/slow interface with different inclined angles were made (Abd-el Fattah, Henderson \& Lozzi 1976; Abd-el Fattah \& Henderson 1978a,b; Henderson, Colella \& Puckett 1991) for more gas combinations such as $\mathrm{CO}_{2} / \mathrm{CH}_{4}$ and air/SF . The transition of refraction pattern from regular to irregular was observed with the incident angle increasing but keeping the strength of the incident shock constant for a fixed gas combination, and different irregular refraction patterns were found for different strengths of the incident shock. For the slow/fast case, the appearance of bound and free precursor shocks is the most remarkable feature in the irregular refraction system and the authors believed that transition will occur from one wave system to another.

During the shock-interface interaction, characteristic vortices will be generated due to the baroclinic mechanism (caused by the misalignment between the pressure and density gradients) and enhance the mixing of the gas inhomogeneity with the ambient gas. Under the action of the vorticity produced, the shape of the interface will be distorted and, ultimately, the fluids will mix with each other thoroughly. This flow instability phenomenon was first studied theoretically and numerically by Richtmyer (1960) and experimentally studied by Meshkov (1969). From then on, the RichtmyerMeshkov instability (RMI) has become increasingly attractive owing to its significant applications in many fields such as inertial confinement fusion (Lindl, McCrory \& Campbell 1992) and supernova explosions (Arnett et al. 1989).

In the experimental study of shock-interface interaction, especially the RMI, it is crucial to generate an ideal initial interface to separate different fluids. The shapes of the gas interface in previous studies mainly include the single-mode interface (Meshkov 1969; Brouillette 2002; Jacobs \& Krivets 2005; Mariani et al. 2008; Long et al. 2009; Balakumar et al. 2012; Luo, Wang \& Si 2013), the spherical or cylindrical bubble formed by a soap film (Haas \& Sturtevant 1987; Hosseini \& Takayama 2005; Layes, Jourdan \& Houas 2009; Haehn et al. 2011, 2012; Ranjan, Oakley \& Bonazza 2011; Zhai et al. 2011; Si et al. 2012), the membrane-less gas cylinder (Jacobs 1992, 1993; Tomkins et al. 2008; Zhai et al. 2014; Zou et al. 2010) or gas curtain (Jacobs et al. 1993; Orlicz et al. 2009; Balakumar et al. 2012; Balasubramanian et al. 2012; Tomkins et al. 2013) formed by the jet technique and the inclined planar interface (Wang et al. 2012; McFarland et al. 2014). Besides these classical interface shapes, Mikaelian (2005) theoretically and numerically studied the RMI on an initial interface with a discontinuous change in its first derivative. Bates, Nikiforakis \& Holder (2007) studied the evolution of a shocked rectangular block generated by a microfilm membrane. Recently in our laboratory, Wang, Si \& Luo (2013) used thin pins to restrict the soap film to form various polygonal interfaces, such as square and triangular interfaces, and investigated the evolution of these shocked air/SF 6 interfaces.

It can be concluded that the interface shapes in shock-interface interaction studies are limited and little attention has been paid to other types of interface such as 
polygonal. The polygonal interface can provide good conditions for shock refraction studies. Therefore, it is expected that the shock-polygonal interface interaction would provide canonical results of shock refraction. It is also of significance to explore the effect of interface shape on the RMI. This is the motivation for the current study. We shall investigate the interaction of a shock wave with a polygonal $\mathrm{N}_{2}$ volume surrounded by $\mathrm{SF}_{6}$.

\section{Experimental and numerical methods}

In this work, the soap film technique developed in our laboratory (Wang et al. 2013) is adopted to form the polygonal interfaces in which thin pins $(0.25 \mathrm{~mm}$ in diameter) are used to connect the adjacent sides of the polygonal soap film at a vertex to avoid the pressure singularity (Isenberg 1992). Specifically, thin pins around the corners of the polygon are first fixed between two parallel Plexiglas plates ( $3 \mathrm{~mm}$ in thickness) in the test section. By blowing a soap bubble (with $\mathrm{N}_{2}$ ) into the test section, the soap film will be perpendicular to the Plexiglas when contacting it due to the surface tension. When the soap film connects all pins, the gas flow into the soap bubble is stopped. At this time, the volume formed is over-expanded due to the overpressure inside and has a circular cylindrical shape. Then, the pressure inside the soap bubble is decreased by pumping out the gas until the soap films connecting the pins become flat (monitored by a video camera). Now an almost ideal two-dimensional polygonal gas interface is formed. Note that during the pumping process some remnants of the soap film are left on the Plexiglas plates as can be seen in the experimental schlieren images.

In our experiments, the test section is first separated from the driven section and is tilted an angle of $\sim 20^{\circ}$ to the horizontal plane. Then $\mathrm{SF}_{6}$ is injected into the test section from its lower side. Because the density of $\mathrm{SF}_{6}$ is much larger than that of air, the air in the test section is exhausted and replaced gradually by $\mathrm{SF}_{6}$. A gas concentration detector is placed at the other side of the test section to ensure the $\mathrm{SF}_{6}$ purity in the test section. Once the concentration of $\mathrm{SF}_{6}$ meets the requirement, the upper side of the test section is sealed with a plastic wrap $(\sim 10 \mu \mathrm{m}$ in thickness) and the test section is placed horizontally and connected to the driven section. In this way, the test section is filled with $\mathrm{SF}_{6}$ and the $\mathrm{N}_{2}$ gas cylinder surrounded by $\mathrm{SF}_{6}$ can be easily formed. In this work, three types of polygonal interface, namely a square, an equilateral triangle and a diamond, are formed, as indicated in figure 1 in which the black points denote the thin pins. The effects caused by the thin pins and fine chamfers at the vertexes used in this technique of interface formation on the shock propagation and interface evolution were evaluated by comparing numerical simulations with shock tube experiments and were found to be very limited as presented in the previous work (Wang et al. 2013).

The experiments are conducted in the same horizontal shock tube as in our previous work (Wang et al. 2013), which consists of a $1.7 \mathrm{~m}$ driver section, a $2.0 \mathrm{~m}$ driven section, a $0.5 \mathrm{~m}$ test section and an end section of $0.7 \mathrm{~m}$ with a rectangular cross-sectional area of $140 \mathrm{~mm} \times 20 \mathrm{~mm}$. The width of the polygonal interfaces, as indicated in figure 1, is smaller than that of the test section $(140 \mathrm{~mm})$. The small height of the test section $(h=20 \mathrm{~mm})$ is adopted to minimize any gravity effect on the test gas and to reduce any three-dimensional effect on the interface. Moreover, under the conditions of the incident shock Mach number $M s=1.29 \pm 0.01$ and the experimental duration of approximately $1 \mathrm{~ms}$, the thickness of the boundary layers on the top and bottom walls of the test section is estimated in the order 

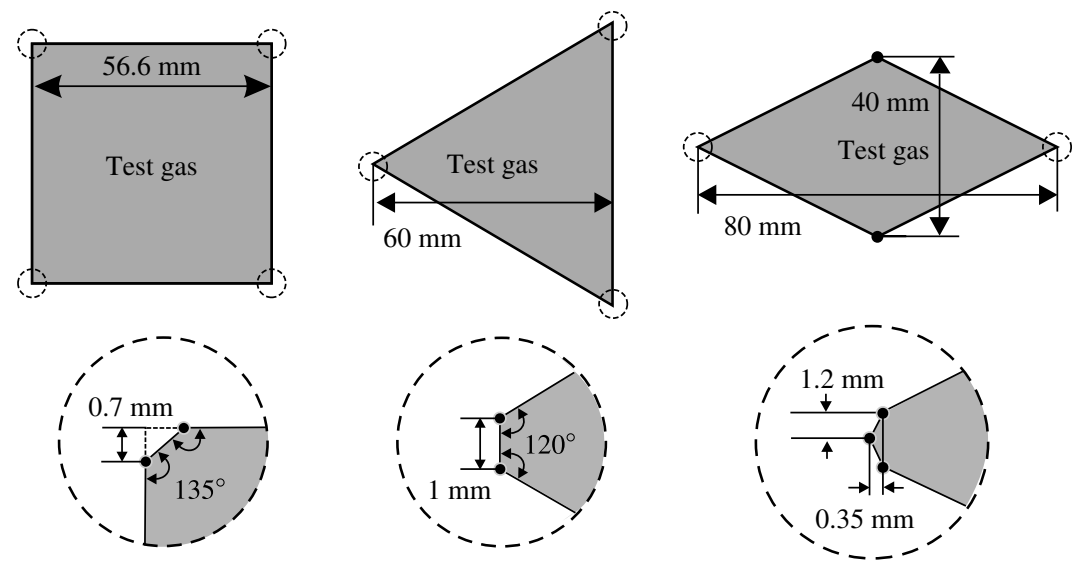

FIGURE 1. Schematic diagrams of the three polygonal interfaces.

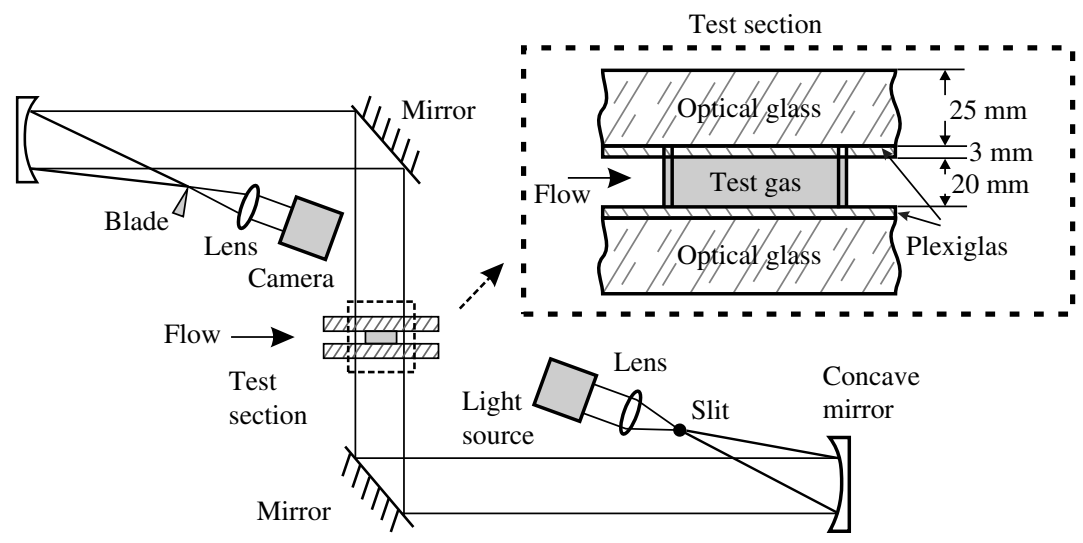

FIGURE 2. Schematic diagrams of the schlieren system and the test section.

of $\sqrt{\mu t / \rho} \sim 0.1 \mathrm{~mm}$ (the viscosity of $\mathrm{N}_{2}$ is $\mu=1.74 \times 10^{-5} \mathrm{~Pa} \mathrm{~s}$ and the density of $\mathrm{N}_{2}$ is $\rho=1.164 \mathrm{~kg} \mathrm{~m}^{-3}$ ), which is far smaller than the height of the shock tube test section. Therefore, the boundary layers on the top and bottom of the test section are thin enough that they have little effect on the depth-integrated images obtained through the schlieren system. Two thin Plexiglas plates are placed on the upper and lower faces of the test chamber as sketched in figure 2. To mount thin pins, through holes are drilled on the upper Plexiglass plate and blind holes are made on the lower plate. Prior to the experiment, thin pins are inserted downwards into the holes of the lower plate through the holes of the upper plate. Two thick optical glass plates $(25.0 \mathrm{~mm}$ in thickness) reinforce the Plexiglas plates from top and bottom of the visualizing windows. The development of shock-accelerated polygonal interfaces, illuminated by a DC regulated light source (DCR III, SCHOTT North America, Inc.), is captured by high-speed schlieren photography as presented in figure 2 . In order to facilitate the light passing through the test section vertically, two parallel planar mirrors with a diameter of $200 \mathrm{~mm}$ are added in the traditional Z-fold schlieren system (Zhai et al. 2011; Si et al. 2012). In the present study, the frame rate of the high-speed video 
camera (FASTCAM SA5, Photron Limited) is up to 37500 f.p.s., the shutter speed of the camera is $1 / 475000 \mathrm{~s}$ and the pixel resolution is $0.34 \mathrm{~mm}$ pixel $^{-1}$.

The numerical method VAS2D (Sun \& Takayama 1999) is adopted to simulate the process of shock-polygonal interface interaction. This method has been well validated for shock-body interaction (Sun \& Takayama 1999), shock-bubble interaction (Zhai et al. 2011) and shock-polygonal heavy interface interaction (Wang et al. 2013). In the numerical algorithm, the governing equations adopt the two-dimensional Euler equations which can be written in vectorial form as

$$
\frac{\partial \boldsymbol{U}}{\partial t}+\frac{\partial \boldsymbol{F}}{\partial x}+\frac{\partial \boldsymbol{G}}{\partial y}=0,
$$

where $\boldsymbol{U}$ represents the conserved variables, and $\boldsymbol{F}$ and $\boldsymbol{G}$ the convective fluxes in the $x$ and $y$ directions, respectively:

$$
\boldsymbol{U}=\left(\begin{array}{c}
\rho \\
\rho u \\
\rho v \\
\rho E \\
\rho_{t}
\end{array}\right), \quad \boldsymbol{F}=\left(\begin{array}{c}
\rho u \\
\rho u^{2}+p \\
\rho u v \\
(\rho E+p) u \\
\rho_{t} u
\end{array}\right), \quad \boldsymbol{G}=\left(\begin{array}{c}
\rho v \\
\rho u v \\
\rho v^{2}+p \\
(\rho E+p) v \\
\rho_{t} v
\end{array}\right),
$$

with $u$ and $v$ being the velocities in the $x$ and $y$ directions, respectively. In the Euler equations, $\rho=\rho_{a}+\rho_{t}$ is the density of the mixture gas with $\rho_{a}$ and $\rho_{t}$ the density of the ambient gas and the test gas, respectively. $E$ is the total energy of the mixture, which is defined in the relation

$$
\rho E=\rho_{a} e_{a}+\rho_{t} e_{t}+\rho\left(u^{2}+v^{2}\right) / 2,
$$

with $e_{a}$ and $e_{t}$ being the internal energy of the ambient gas and the test gas, respectively. The pressure $p$ is related to $\rho$ by the perfect gas law $p=\rho R T$ with $R$ the gas constant for the mixture and $T$ the temperature.

The VAS2D method uses the finite volume method and has a second-order precision in both temporal and spatial scales based on the MUSCL-Hancock approach. An unstructured adaptive mesh is employed such that it refines local complex areas and can effectively capture waves and interface evolutions in compressible flows. The description of the computational domain, the initial boundary conditions and the grid study can be found in our previous work (Wang et al. 2013). According to the grid study, a uniform initial mesh size of $0.2 \mathrm{~mm}$ is employed and a finer mesh of $0.05 \mathrm{~mm}$ is used where greater density gradients exist. Note that because of experimental difficulties it is nearly impossible to ensure that the gases both inside and outside the polygonal cylinders are pure. The gas contamination must be considered in evaluating the experimental data. In order to better determine the actual gas concentrations in both regions, the velocities of shocks inside and outside the volume are first experimentally measured and then the one-dimensional gas dynamics theory is used to solve the problem of a planar shock impinging on a planar or an inclined gas interface for each case. In this way, the gas concentrations in both regions can be calculated, and the actual gas properties used in the computation are determined as listed in table 1 . 


$\begin{array}{lcccc}\text { Case } & \begin{array}{c}\text { Interior } \\ \text { gas }\end{array} & \begin{array}{c}\text { Molecular weight } \\ \left(\mathrm{g} \mathrm{mol}^{-1}\right)\end{array} & \begin{array}{c}\text { Density } \\ \left(\mathrm{kg} \mathrm{m}^{-3}\right)\end{array} & \begin{array}{c}\text { Sound speed } \\ \left(\mathrm{m} \mathrm{s}^{-1}\right)\end{array} \\ \text { Square } & 100 \% \mathrm{~N}_{2} & 28 & 1.14 & 352 \\ \text { Triangle } & 86 \% \mathrm{~N}_{2}+14 \% \mathrm{SF}_{6} & 44.5 & 1.81 & 279.2 \\ \text { Diamond } & 94 \% \mathrm{~N}_{2}+6 \% \mathrm{SF}_{6} & 35.1 & 1.43 & 314.5\end{array}$

TABLE 1. Physical properties of actual test gas inside the volume in numerical simulations at $T_{0}=298 \mathrm{~K}$ and $p_{0}=101325 \mathrm{~Pa}$. Here, $m \% \mathrm{~A}+n \% \mathrm{~B}$ denotes that the gas used is the mixture of $m \% \mathrm{~A}$ and $n \% \mathrm{~B}$ (the mole fraction). In all cases, the ambient gas is considered as pure.

\section{Interface morphology and features}

The experimental and numerical results of a planar shock interacting with the polygonal interface are presented and compared in figures $3-5$. The initial time is defined as when the incident shock collides with the leftmost part of the interface for all cases. Note that the circular 'wavelike' structures around the initial polygonal shape in the first photograph before the shock wave arrival are the remnants of the soap film on the observation windows from blowing the soap bubble in the test section.

Figure 3 presents the evolution of the square interface impacted by a planar shock wave $(M s=1.28)$. With the incident shock (is) travelling along the upper and lower boundaries, an irregular refraction happens owing to the nearly glancing incidence (the incident angle $\theta \approx 90^{\circ}$ ) where the transmitted shock $(t s)$ moves along the interface faster than the $i s$. The $t s$ is itself refracted at the interface and transmits a new shock (free precursor shock $f p s$ ) into the ambient gas $(t=82 \mu \mathrm{s})$. Then, the $f p s$ and $i s$ meet and mutually modify each other, forming a triple point $(t p)$, a Mach stem $(m s)$ and a shock $n$ outside the volume, as indicated at $t=188 \mu \mathrm{s}$. When the $t s$ inside the volume encounters the downstream interface, a secondary transmitted shock (sts) travelling downwards and a reflected shock propagating upwards are generated $(t=188 \mu \mathrm{s})$. Due to the vorticity deposition on the interface, two vortices are generated at the left corners. At $t=374 \mu \mathrm{s}$, a diffracted shock $(d s)$ is generated, travelling along the rightmost surface, and the reflected shock $(r t s)$ is transmitted at the upstream surface. The shocks reflected from the shock tube wall (wrs) are also clearly seen at $t=588 \mu \mathrm{s}$, which may influence the interface height and the vorticity production. As time proceeds, the influence of the shock wave on the interface evolution is lessened and the vortex pair at the corners grows gradually, induced by the vorticity produced $(t=588-1334 \mu \mathrm{s})$. At later times, the flow field is fully governed by the vortex pair $(t=1574 \mu \mathrm{s})$.

The interface morphology of the equilateral triangular interface accelerated by a planar shock wave $(M s=1.29)$ is illustrated in figure 4. Similar to the square case, the velocity of the $t s$ moving along the interface is larger than that of the $i s$, and the appearance of the fps is the remarkable feature $(t=156 \mu \mathrm{s})$. The $t s$ has a circular arc shape and is nearly at glancing incidence to the interface. Meanwhile, a reflected rarefaction wave ( $r r w)$ arises from the interface surface. Shortly after the $i s$ contacts the interface, a phase reversal process occurs where a small ' $\mathrm{SF}_{6}$ jet' is generated at the apex of the interface. Later, the secondary reflection and transmission arise when the $t s$ meets the downstream boundary $(t=264 \mu \mathrm{s})$ and in the subsequent development, the sts, $m s, d s, r t s$ and $w r s$ are also observed in the flow field. As time elapses, the jet grows constantly and the distance between the jet head and the 


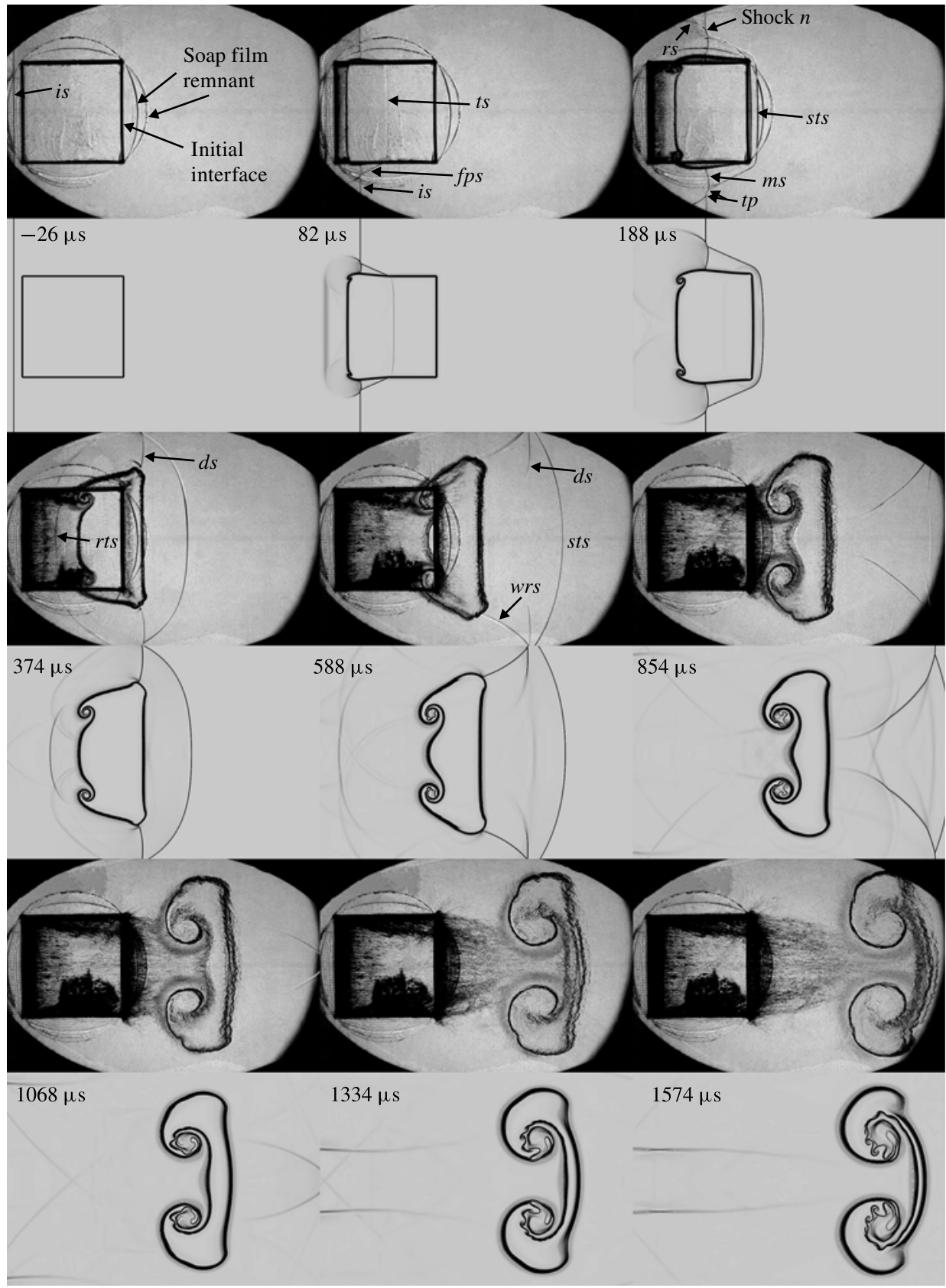

FIGURE 3. Experimental (upper) and numerical (lower) schlieren images of a square $\mathrm{N}_{2}$ volume surrounded by $\mathrm{SF}_{6}$ impacted by a planar shock wave $(M s=1.28)$. is, incident shock; $t s$, transmitted shock; fps, free precursor shock; $r s$, reflected shock; $t p$, triple point; $m s$, Mach stem; sts, secondary transmitted shock; $d s$, diffracted shock; rts, reflected transmitted shock; wrs, reflected shock from the wall.

downstream boundary becomes shorter $(t=156-450 \mu \mathrm{s})$. The connection between the two vortices of the jet becomes thinner $(t=636-1250 \mu \mathrm{s})$ and eventually breaks up, forming two independent vortices $(t=1516 \mu \mathrm{s})$. 


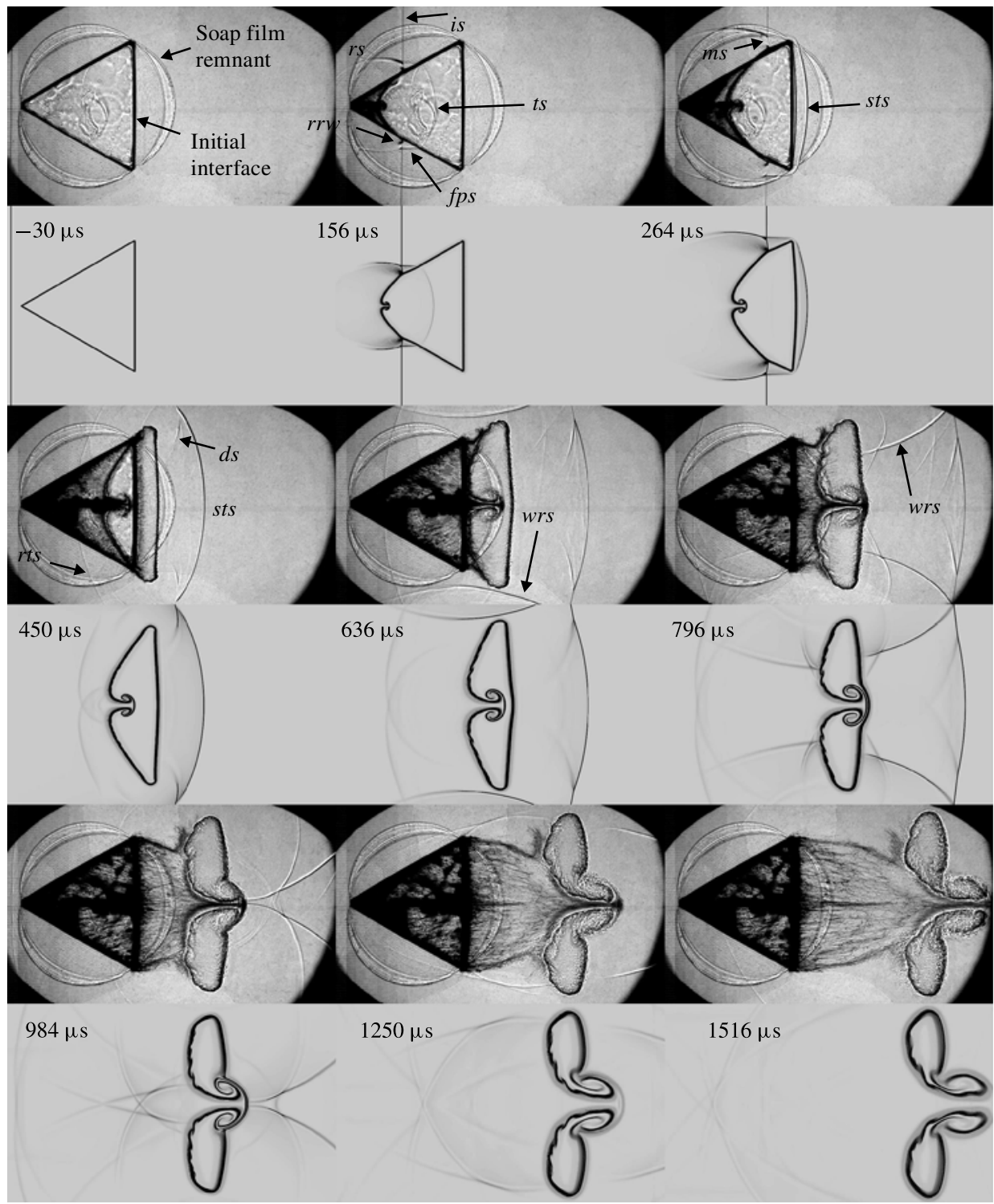

FIGURE 4. Experimental and numerical schlieren images of a triangular $\mathrm{N}_{2}$ volume surrounded by $\mathrm{SF}_{6}$ impacted by a planar shock wave $(M s=1.29)$. $r r w$, reflected rarefaction waves, the other symbols are the same as those indicated in figure 3.

The development of the diamond interface induced by a planar shock wave $(M s=1.30)$ is shown in figure 5. Similar phenomena, including the wave motions and the interface evolution, to the triangular interface, occur for the left half of the diamond interface owing to the similar geometry $(t=122-228 \mu \mathrm{s})$. After the shocks pass through the vertexes at the top and bottom as well as the rightmost part of the interface, small vortical structures, though not evident, are generated at these places. The phase reversal phenomenon also happens and the ' $\mathrm{SF}_{6}$ jet' generated moves faster than the upstream interface $(t=362-814 \mu \mathrm{s})$. Ultimately, the jet catches up with and 


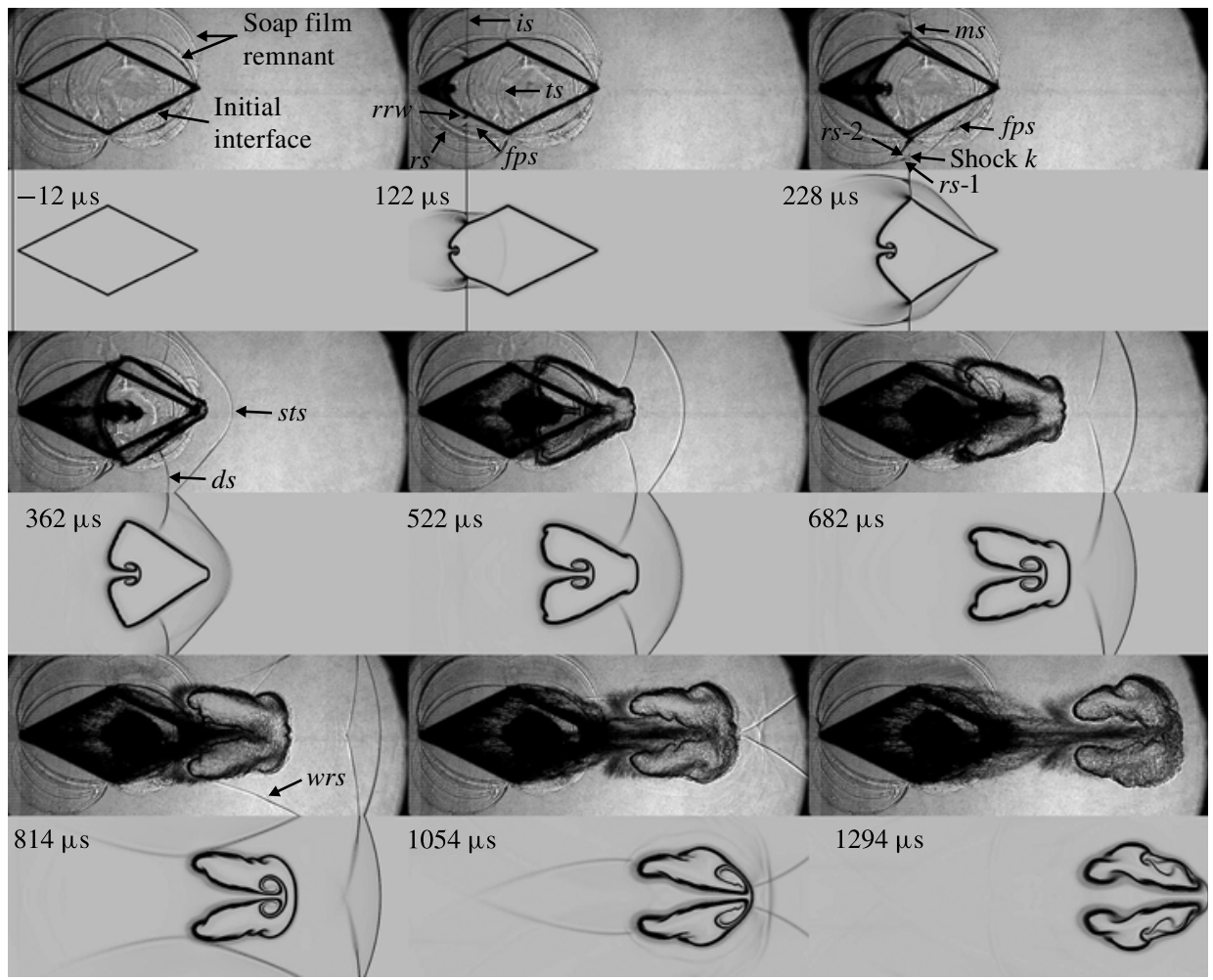

FIgURE 5. Experimental and numerical schlieren images of a diamond $\mathrm{N}_{2}$ volume surrounded by $\mathrm{SF}_{6}$ impacted by a planar shock wave $(M s=1.30)$. $r s-1$, reflected shock $1 ; r s-2$, reflected shock 2, the other symbols are the same as those indicated in figure 3 .

breaks through the downstream interface, resulting in the formation of two vortices $(t=1054-1294 \mu \mathrm{s})$.

Quantitatively, the movement of the distorted interface, and the length and height of the interface structures are measured from experiments and computations as shown in figure 6 and a schematic of measuring position is also indicated in each figure. For all three types of interface, the upstream interfaces, $X_{l}$, move with nearly constant velocities $\left(\sim 71, \sim 78\right.$ and $\sim 85 \mathrm{~m} \mathrm{~s}^{-1}$ for square, triangular and diamond interfaces, respectively). The downstream interfaces, $X_{r}$, are stationary before the shock passes through them and subsequently they also move with nearly constant velocities $\left(\sim 72, \sim 87\right.$ and $\sim 84 \mathrm{~m} \mathrm{~s}^{-1}$ for square, triangular and diamond interfaces, respectively). Rudinger \& Somers (1960) proposed a simple model to predict the velocity of inhomogeneity by assuming that the inhomogeneity has fully developed into a vortex pair. In their model, the velocity of the vortex pair $\left(u_{B}\right)$ is related to the flow velocity $\left(u_{G}\right)$ behind the $i s$ by $u_{B}=u_{G}\left(1-0.203 A_{t}\right)$, where $A_{t}$ is the Atwood number defined as $A_{t}=\left(\rho_{t}-\rho_{a}\right) /\left(\rho_{t}+\rho_{a}\right)$. According to this expression and the values listed in table 1 , the velocities of vortex pairs are predicted to be 73,74 and $77 \mathrm{~m} \mathrm{~s}^{-1}$ for the square, triangular and diamond cases, respectively. Because the upstream and downstream boundaries move with a nearly constant velocity in all three cases during the time studied, the experimentally measured velocities of the upstream or downstream interfaces can reflect the velocity of the vortex pair. It can 

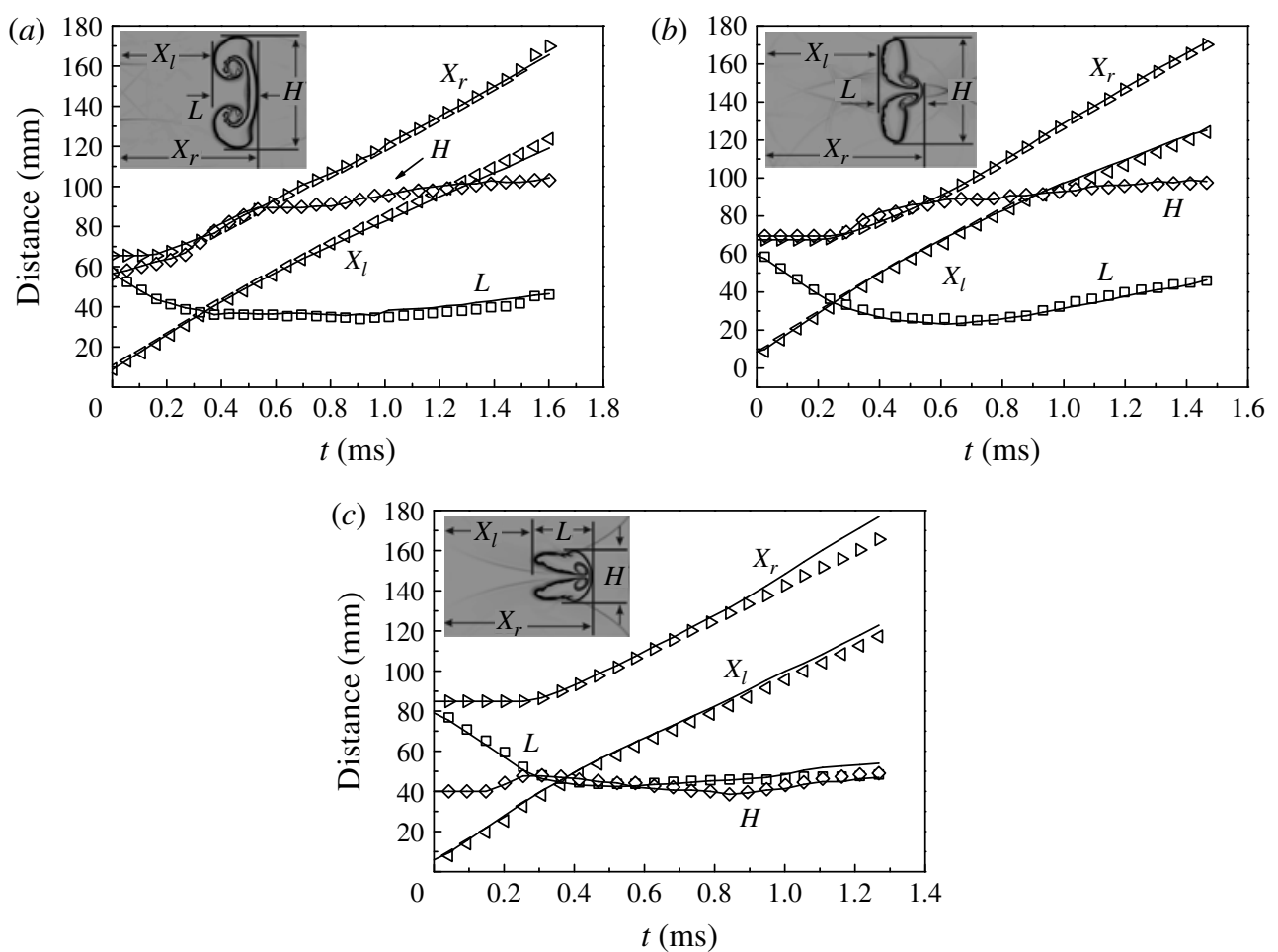

FIGURE 6. The movement of the distorted upstream and downstream interfaces, and the interface length and height for the square $(a)$, the triangle $(b)$, and the diamond $(c)$ from experiments and computations. The symbols indicate experimental results and the lines denote the numerical ones.

be found from the comparison that the theoretical values agree with the experimental ones, especially for the square case, which is because the square interface has evolved into a vortex pair after a certain time (approximately $1000 \mu \mathrm{s}$ after the is impact), while the triangular and diamond interfaces have not during the time studied.

The interface length $L$, defined as $X_{r}-X_{l}$, decreases due to the shock compression at early stage for all cases. For the square case, after the compression phase, the velocities of upstream and downstream interfaces are similar and $L$ is almost constant for a long evolving time, as shown in figure $6(a)$. For the triangular case, after the ' $\mathrm{SF}_{6}$ jet' penetrates through the downstream interface $(\sim 0.85 \mathrm{~ms})$, a faster movement of the downstream interface is observed because $X_{r}$ thereafter denotes the jet head. The interface length therefore develops with a small increase, as presented in figure 6(b). Similar movements of the upstream and downstream interfaces after the compression phase for the diamond case also result in the steady evolution of the interface length, as indicated in figure 6(c). When the ' $\mathrm{SF}_{6}$ jet' penetrates through the downstream interface $(\sim 1.05 \mathrm{~ms})$, it is predicted that the interface length for the diamond case will increase as well.

Owing to the continuous rotation of the vortex pair, the interface height $H$ increases constantly for the square case until the reflected shock waves from the wall strike the interface $(\sim 0.55 \mathrm{~ms})$, reducing the growth rate of the interface height. For the triangular interface, before the $t s$ traverses the downstream boundary $(\sim 0.16 \mathrm{~ms})$, 
(a)

$(c)$
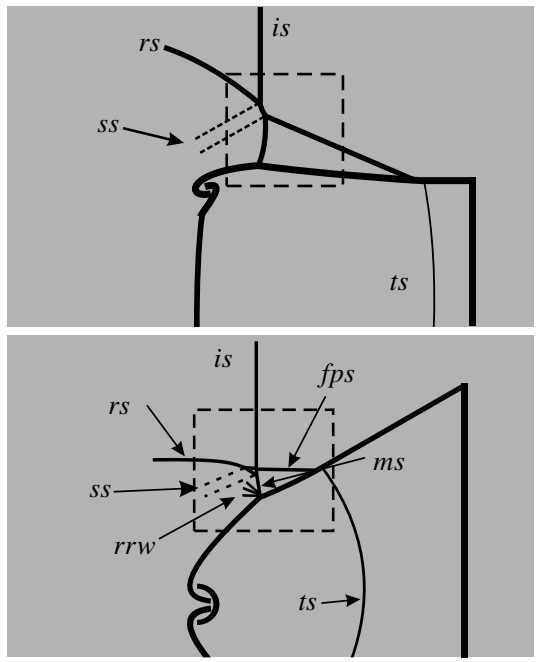

(b)

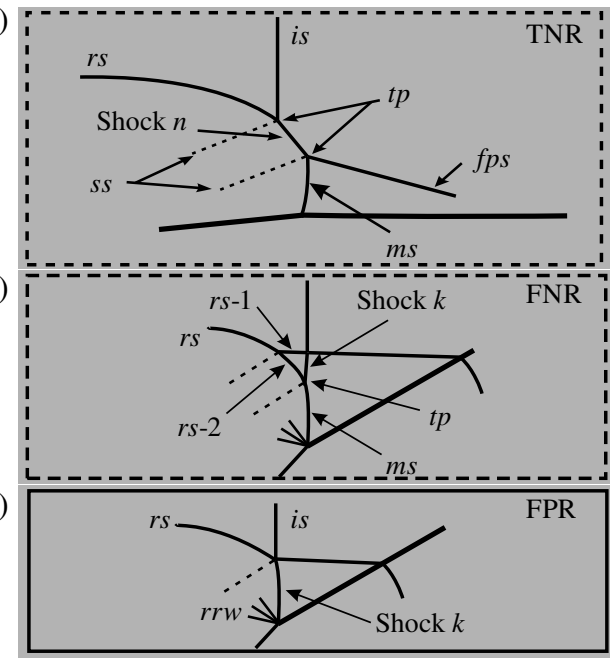

FIGURE 7. The wave patterns of a shock interacting with an $\mathrm{N}_{2}$ volume surrounded by $\mathrm{SF}_{6}$ : (a) square cylinder at $t=180 \mu \mathrm{s}$ and $(b)$ the enlargement of the details of TNR; (c) triangular cylinder at $t=212 \mu \mathrm{s}$ and the enlargement of the details of $(d) \mathrm{FNR}$ as well as (e) FPR. ss denotes the slip surface, and the other symbols are the same as those indicated in figure 3 .

the height remains constant. Subsequently, the height increases due to the stretching of the downstream boundary in the vertical direction under the action of the shock wave, and then the growth rate is decreased because of the wrs compression at $t \sim 0.65 \mathrm{~ms}$. For the diamond interface, however, shortly after a transient increase, the interface height experiences a decrease because the upper and lower boundaries are driven towards the centre by the fast-moving ' $\mathrm{SF}_{6}$ jet'. This trend continues until the measuring position is changed when the height of the upstream interface is larger than that of the downstream interface at $t \sim 0.95 \mathrm{~ms}$.

It can be concluded that the initial shape of the interface has a significant effect on the interface morphology and features. It can be easily seen from the qualitative and quantitative comparisons that the experimental and numerical results are in good agreement, especially at an early stage and a small discrepancy arises at a late stage due to the diffusion of the interface and mixing between fluids that result in an indistinct interface boundary.

\section{Wave patterns and characteristics}

The type of shock refraction at a gaseous interface depends on the incident angle $\theta$ if the strength of is is held constant. With $\theta$ increasing, a series of flow patterns, from regular refraction to all kinds of irregular refraction, will be observed. In the study of Abd-el Fattah \& Henderson (1978b), the refraction phenomena at a slow/fast interface were classified into three groups (very weak, weak and strong) characterized by different strengths of the $i s$, assuming that the gases were nearly perfect. In the present study, the strengths of the $i$ s are almost equal for the three cases and the incident angles for the hypotenuse of the triangle, the upstream hypotenuse of the diamond and the upper or lower surface of the square interface, are $60^{\circ}, 63.4^{\circ}$ and $90^{\circ}$, respectively. Under the condition of such large incident angles, only irregular refraction phenomena can be found in the present work. 
Figure $7(a, b)$ shows the wave pattern at an early stage of the interaction of the shock with the square cylinder. Around the upper side of the interface, the $t s$ moves along the interface faster than the is does, and transmits a fps into the ambient gas. Under the interaction of the $i s$ and the $f p s$, a shock $n$, an $m s$ and two $t p$ appear. This type of refraction of a shock wave at a slow/fast interface is known as 'twin von Neumann refraction' (TNR) (Abd-el Fattah \& Henderson 1978b). Figure 7(c,d) presents the wave system at an early stage of the interaction of the shock with the triangular cylinder. This type of refraction of a shock wave at a slow/fast interface is referred to as 'free precursor von Neumann refraction' (FNR) (Abd-el Fattah \& Henderson 1978b), which is thought of as a combination of the $t s$, a fps and a Mach reflection. One can observe that FNR is quite similar to another wave system called 'free precursor refraction' (FPR) (Jahn 1956; Abd-el Fattah et al. 1976; Abd-el Fattah \& Henderson 1978b), as sketched in figure 7(e). Abd-el Fattah \& Henderson (1978b) indicated that the difference between FPR and FNR is that in FPR the shock $k$ propagates to the interface undisturbed, whereas it undergoes a von Neumann reflection in FNR as it approaches the interface. They also pointed out that the property which determines whether a free precursor system is FPR or FNR is whether the flow downstream of the shock $k$, indicated in figure $7(d, e)$, is supersonic or subsonic at the interface, and further they defined this to be the difference between the 'weak' and 'very weak' groups.

It can be found from the previous results (Abd-el Fattah \& Henderson 1978b) that FNR was observed in the 'very weak' group and FPR and TNR in the 'weak' group at a $\mathrm{CO}_{2} / \mathrm{CH}_{4}$ interface. TNR has never been observed for any incident angle in the 'very weak' group. In other words, neither FNR nor TNR has ever been observed at a slow/fast interface within the same group with any incident angle in the previous work. However, in the present work, FNR is observed at the triangular interface and TNR at the square interface, and both wave patterns are found with similar strengths of the is. Compared with the previous work, this observation indicates that the wave pattern of a shock refraction at a density interface will be strongly affected by the gas combination. Note that the gas concentrations for the square and triangular cases in the present work are different, which may also have an influence on the shock refraction phenomenon.

It is known that TNR is derived from FPR with $\theta$ increasing. It is also believed that FNR would be converted into FPR under certain conditions (Abd-el Fattah \& Henderson 1978b). But, unfortunately, there is no experimental support for this. Based on this consideration, FNR and TNR may occur within the same group if some requirements are met as Abd-el Fattah \& Henderson (1978a) declared that the wave system could apparently be suppressed by paying attention to gas purity and boundary conditions. Jahn (1956) also thought that various irregular patterns were related to each other in a continuous sequence, and they may be considered to evolve from one to another. The transition of the wave pattern from FNR to FPR and later to TNR is observed experimentally and numerically in the shock-diamond interface interaction as shown in figure 8. The only difference of the wave pattern in the present work with the previous work is that the fps in the present work has already moved away from the interface boundary. Therefore, our results confirm the transition viewpoints proposed in the previous work and provide direct experimental evidence for that.

Based on the high-speed images, the velocities of the $t s$ along the central line of the interface $(t s-c)$ and the boundary $(t s-b)$ are measured to be 282.5 and $274.5 \mathrm{~m} \mathrm{~s}^{-1}$ respectively. Actually the $t s$ near the interface has weakened into an evanescent wave 
(a)

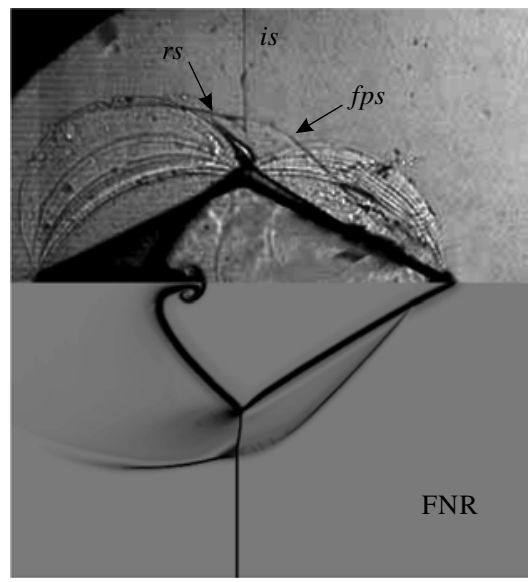

(c)

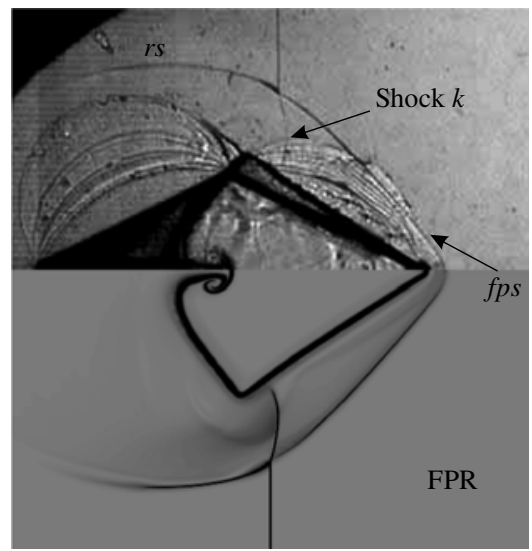

(b)

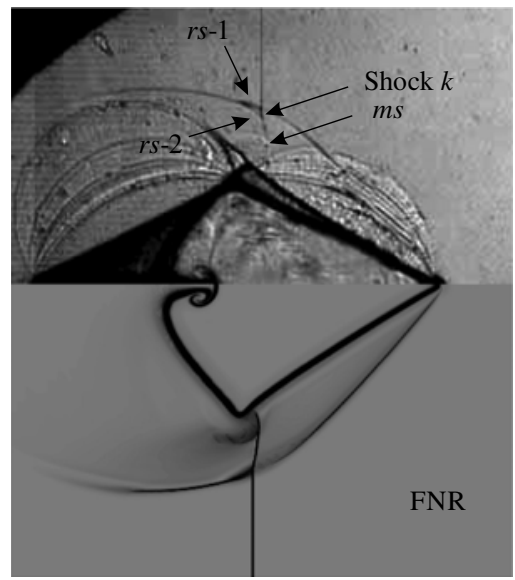

(d)

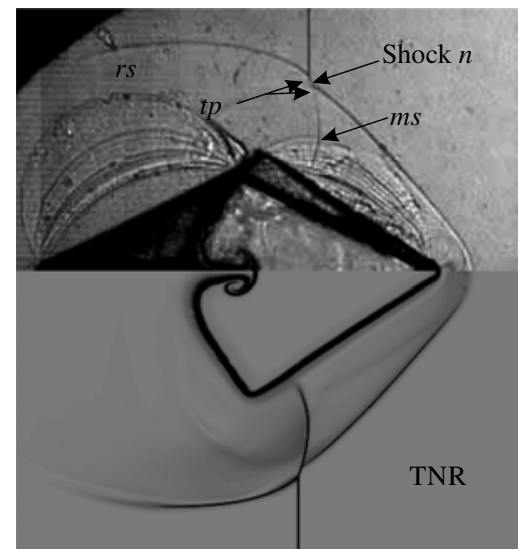

FIGURE 8. The transition of wave patterns from FNR at $(a) t=228 \mu \mathrm{s}$ and $(b) t=254 \mu \mathrm{s}$, to FPR at $(c) t=281 \mu \mathrm{s}$, and later to TNR at $(d) t=308 \mu \mathrm{s}$, of a shock interacting with a diamond $\mathrm{N}_{2}$ volume surrounded by $\mathrm{SF}_{6}$. The upper and lower images are from experiment and computation, respectively. The symbols are the same as those indicated in figure 3 .

since the sound speed inside the volume is $279 \mathrm{~m} \mathrm{~s}^{-1}$. The same conclusion was drawn by Jahn (1956) in an experiment even though there were no quantitative data to prove it. Moreover, Henderson et al. (1991) found from their numerical results that the $t s$ is a locally evanescent wave but their experiments could not resolve it. The results in the present work provide the experimental evidence for this standpoint. A similar analysis for the diamond interface is conducted and the same conclusion is reached where the values of $t s-\mathrm{c}$ and $t s-\mathrm{b}$ are measured to be 318.3 and $308.2 \mathrm{~m} \mathrm{~s}^{-1}$ respectively (the sound speed inside the volume is $314.5 \mathrm{~m} \mathrm{~s}^{-1}$ ). For the square interface, the velocities of $t s-\mathrm{c}$ and $t s-\mathrm{b}$, the $i s$, as well as the two $t p$ are experimentally acquired as indicated in figure $9(a)$. Once more, a discrepancy is observed between the velocities of $t s-\mathrm{c}$ and $t s-\mathrm{b}$ and these two values are found to be 357.5 and $338.5 \mathrm{~m} \mathrm{~s}^{-1}$, respectively (the sound speed inside the volume is $352 \mathrm{~m} \mathrm{~s}^{-1}$ ). In addition, the two tp are considered to have originated from the interface vertex, and the time variations of the angles between the original horizontal boundary and the trajectories of the two tp are given in figure $9(b)$. It can be seen 

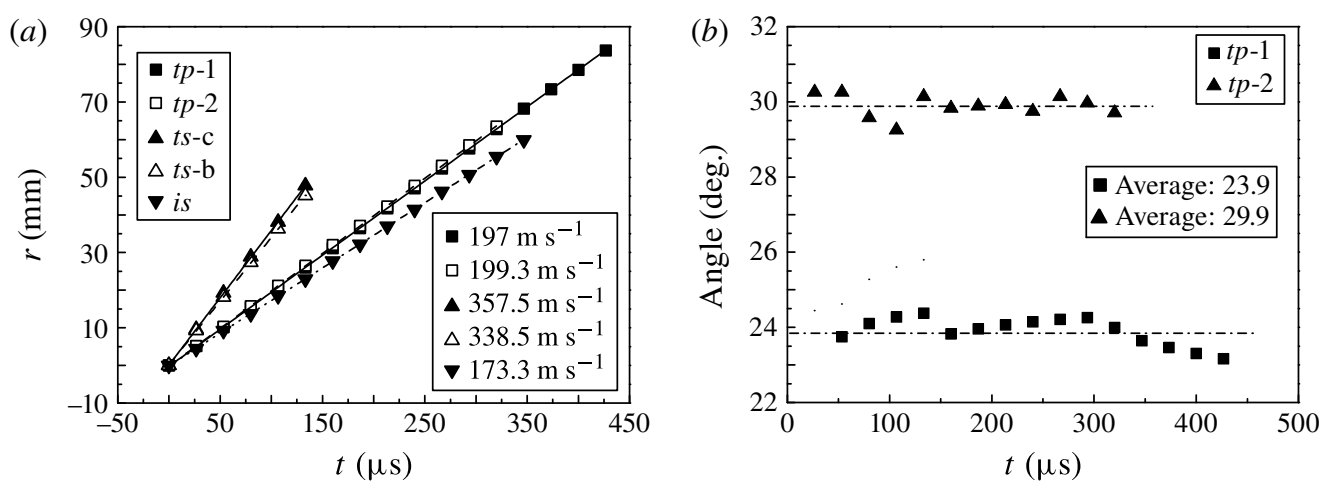

FIgURE 9. (a) Displacement histories of the shocks and triple points, and $(b)$ time variation of the angle between the horizontal boundary and the trajectories of two triple points, for the square interface. $t s-\mathrm{c}$, the transmitted shock along the central line; $t s-\mathrm{b}$, the transmitted shock along the boundary; $t p-1$, the first triple point; $t p-2$, the second triple point.

that the two tp travel along a nearly straight line with a quite similar velocity which means that the motion of the $t p$ is self-similar or pseudo-stationary.

\section{Conclusions}

The interaction of a planar shock with a polygonal $\mathrm{N}_{2}$ volume surrounded by $\mathrm{SF}_{6}$ is investigated by experiments and numerical simulations. The emphasis is on the wave pattern of the shock refraction at the slow/fast interface and on the effect of the interface shape on the interface morphology.

Three different interface configurations, namely a square, a diamond and a triangle, are formed through the restriction of the soap film by thin pins. Distinct schlieren images of the evolutions of the interfaces induced by a planar shock are obtained from both numerical simulations and experiments, and good agreement is achieved between them. The variations of the interface features, including the interface length and height and the velocities of the distorted interfaces, are compared and good agreements are achieved between experimental and numerical results. It is found that the evolution of these polygonal interfaces is closely related to their initial shapes. In the square interface, two vortices are generated shortly after the shock impact around the left corner and grow with time, and finally dominate the flow field. In the triangular and diamond cases, the small ' $\mathrm{SF}_{6}$ jet' is the most remarkable feature, which grows constantly with time. As time proceeds, the distance between the jet head and the downstream boundary becomes shorter and eventually the jet head penetrates the downstream boundary, forming two independent vortices. These distinct morphologies of the three polygonal interfaces also lead to the different behaviours of the interface features including the length and height. It is also found that the velocities of the vortex pair predicted from the theory of Rudinger \& Somers (1960) agree with the experimental ones, especially for the square case.

The emphasis is then placed on the shock refraction at the slow/fast interface. It is found that the irregular wave patterns of FNR, FPR and TNR all occur at an $\mathrm{SF}_{6} / \mathrm{N}_{2}$ interface for a similar is strength and transitions among them are directly observed from the experiment and computation, which confirms some viewpoints on the wave 
transition raised in the literature, and for the first time provides direct experimental evidence of this. Further, the velocities of the characteristic waves are measured and the $t s$ near the interface is found to be weakened into an evanescent wave. Moreover, the trajectories and velocities of two tp are obtained for the square interface and it is proved that the motion of the $t p$ is self-similar or pseudo-stationary.

The present results can be used to validate the numerical codes or theoretical models for shock-interface interactions, and also enrich the database of interface morphology and wave patterns in shock-interface studies. It is also expected that the effect of interface shape on the development of RMI can be investigated by employing the current method of interface formation. In a following work, advanced flow diagnostic techniques will be applied and more cases of different shapes and test gases will be studied. Furthermore, the interaction of a planar shock wave with a polygonal heavy gas cylinder will be studied experimentally and numerically. In this circumstance, both regular and irregular refraction phenomena will be observed when the incident shock propagates along the interface outside the volume. However, more complex wave patterns, including transmitted shocks, refracted shocks, Mach stems, other shocks and the interactions among them, will be found inside the volume.

\section{Acknowledgements}

This work is supported by the National Natural Science Foundation of China (11272308, 11302219), the Fundamental Research Funds for Central Universities (WK2090050020, WK2320000020) and the China Postdoctoral Science Foundation (BH2090050031).

\section{REFERENCES}

Abd-el Fattah, A. M. \& Henderson, L. F. $1978 a$ Shock waves at a fast-slow gas interface. J. Fluid Mech. 86, 15-32.

Abd-El Fattah, A. M. \& Henderson, L. F. $1978 b$ Shock waves at a slow-fast gas interface. J. Fluid Mech. 89, 79-95.

Abd-el Fattah, A. M., Henderson, L. F. \& LozZi, A. 1976 Precursor shock waves at a slow-fast gas interface. J. Fluid Mech. 76, 157-176.

Arnett, W. D., Bahcall, J. N., Kirshner, R. P. \& Woosley, S. E. 1989 Supernova 1987A. Annu. Rev. Astron. Astrophys. 27, 629-700.

Balakumar, B. J., Orlicz, G. C., Ristorcelli, J. R., Balasubramanian, S., Prestridge, K. P. \& Tomkins, C. D. 2012 Turbulent mixing in a Richtmyer-Meshkov fluid layer after reshock: velocity and density statistics. J. Fluid Mech. 696, 67-93.

Balasubramanian, S., Orlicz, G. C., Prestridge, K. P. \& Balakumar, B. J. 2012 Experimental study of initial condition dependence on Richtmyer-Meshkov instability in the presence of reshock. Phys. Fluids 24, 034103.

Bates, K. R., Nikiforakis, N. \& Holder, D. 2007 Richtmyer-Meshkov instability induced by the interaction of a shock wave with a rectangular block of $\mathrm{SF}_{6}$. Phys. Fluids 19, 036101.

Brouillette, M. 2002 The Richtmyer-Meshkov instability. Annu. Rev. Fluid Mech. 34, 445-468.

HaAs, J. F. \& StURTEvant, B. 1987 Interaction of weak shock waves with cylindrical and spherical gas inhomogeneities. J. Fluid Mech. 181, 41-76.

Haehn, N., Ranjan, D., Weber, C., Oakley, J., Rothamer, D. \& Bonazza, R. 2012 Reacting shock bubble interaction. Combust. Flame 159, 1339-1350.

Haehn, N., Weber, C., Oakley, J. G., Anderson, M. H., Ranjan, D. \& Bonazza, R. 2011 Experimental investigation of a twice-shocked spherical gas inhomogeneity with particle image velocimetry. Shock Waves 21, 225-231. 
Henderson, L. F. 1966 The refraction of a plane shock wave at a gas interface. J. Fluid Mech. 26, 607-637.

Henderson, L. F., Colella, P. \& Puckett, E. G. 1991 On the refraction of shock waves at a slow-fast gas interface. J. Fluid Mech. 224, 1-27.

Hosseini, S. H. R. \& TAKAYAMA, K. 2005 Experimental study of Richtmyer-Meshkov instability induced by cylindrical shock waves. Phys. Fluids 17, 084101.

Isenberg, C. 1992 The Science of Soap Films and Soap Bubbles. Dover.

JACOBS, J. W. 1992 Shock-induced mixing of a light-gas cylinder. J. Fluid Mech. 234, 629-649.

JACOBS, J. W. 1993 The dynamics of shock accelerated light and heavy gas cylinders. Phys. Fluids A 5, 2239-2247.

Jacobs, J. W., Klein, D. L., Jenkins, D. G. \& Benjamin, R. F. 1993 Instability growth patterns of a shock-accelerated thin fluid layer. Phys. Rev. Lett. 70, 583-589.

Jacobs, J. W. \& Krivets, V. V. 2005 Experiments on the late-time development of single-mode Richtmyer-Meshkov instability. Phys. Fluids 17, 034105.

JAHN, R. G. 1956 The refraction of shock waves at a gaseous interface. J. Fluid Mech. 1, 457-489.

Layes, G., Jourdan, G. \& HouAs, L. 2009 Experimental study on a plane shock wave accelerating a gas bubble. Phys. Fluids 21, 074102.

Lindl, J. D., McCrory, R. L. \& CAmpbell, E. M. 1992 Progress toward ignition and burn propagation in inertial confinement fusion. Phys. Today 45, 32-40.

Long, C. C., Krivets, V. V., Greenough, J. A. \& Jacobs, J. W. 2009 Shock tube experiments and numerical simulation of the single-mode, three-dimensional Richtmyer-Meshkov instability. Phys. Fluids 21, 114104.

LuO, X., WANG, X. \& SI, T. 2013 The Richtmyer-Meshkov instability of a three-dimensional air/SF 6 interface with a minimum-surface feature. J. Fluid Mech. 722, R2.

Mariani, C., Vanderboomgaerde, M., Jourdan, G., Souffland, D. \& Houas, L. 2008 Investigation of the Richtmyer-Meshkov instability with stereolithographed interfaces. Phys. Rev. Lett. 100, 254503.

McFarland, J., Reilly, D., Creel, S., McDonald, C., Finn, T. \& Ranjan, D. 2014 Experimental investigation of the inclined interface Richtmyer-Meshkov instability before and after reshock. Exp. Fluids 55, 1640-1653.

Meshrov, E. E. 1969 Instability of the interface of two gases accelerated by a shock wave. Fluid Dyn. 4, 101-104.

Mikaelian, K. O. 2005 Richtmyer-Meshkov instability of arbitrary shapes. Phys. Fluids 17, 034101.

Orlicz, G. C., Balakumar, B. J., Tomkins, C. D. \& Prestridge, K. P. 2009 A Mach number study of the Richtmyer-Meshkov instability in a varicose, heavy-gas curtain. Phys. Fluids 21, 064102.

Ranjan, D., OAKley, J. \& Bonazza, R. 2011 Shock-bubble interactions. Annu. Rev. Fluid Mech. 43, 117-140.

RichtmyeR, R. D. 1960 Taylor instability in shock acceleration of compressible fluids. Commun. Pure Appl. Maths 13, 297-319.

Rudinger, G. \& Somers, L. M. 1960 Behaviour of small regions of different gases carried in accelerated gas flows. J. Fluid Mech. 7, 161-176.

Si, T., ZHAI, Z., LuO, X. \& YANG, J. 2012 Experimental studies of reshocked spherical gas interfaces. Phys. Fluids 24, 054101.

Sun, M. \& TAKayama, K. 1999 Conservative smoothing on an adaptive quadrilateral grid. J. Comput. Phys. 150, 143-180.

Tomkins, C. D., Balakumar, B. J., Orlicz, G. C., Prestridge, K. P. \& Ristorcelli, J. R. 2013 Evolution of the density self-correlation in developing Richtmyer-Meshkov turbulence. J. Fluid Mech. 735, 288-306.

Tomkins, C. D., Kumar, S., Orlicz, G. C. \& Prestridge, K. P. 2008 An experimental investigation of mixing mechanisms in shock-accelerated flow. J. Fluid Mech. 611, 131-150.

WANG, M., SI, T. \& LUO, X. 2013 Generation of polygonal gas interfaces by soap film for RichtmyerMeshkov instability study. Exp. Fluids 54, 1427-1435. 
Wang, T., LiU, J. H., BAI, J. S., JiAng, Y., LI, P. \& LiU, K. 2012 Experimental and numerical investigation of inclined air/SF 6 interface instability under shock wave. Appl. Math. Mech. 33, $37-50$.

ZhaI, Z., SI, T., LUO, X. \& YAng, J. 2011 On the evolution of spherical gas interfaces accelerated by a planar shock wave. Phys. Fluids 23, 084104.

ZhAI, Z., ZHANG, F., SI, T. \& LUO, X. 2014 Evolution of heavy gas cylinder under reshock conditions. J. Vis. 17, 123-129.

Zou, L. Y., LiU, C. L., TAN, D. W., HuAnG, W. B. \& LuO, X. 2010 On interaction of shock wave with elliptic gas cylinder. J. Vis. 13, 347-353. 